\title{
Contents of This Volume
}

\author{
Mike Boyle
}

Published online: 5 March 2013

(C) Springer Science+Business Media Dordrecht 2013

This is a volume focused on symbolic dynamics, to which Kim and Roush contributed so much. The biographical and review articles are evident from their titles. The research papers reflect both recurring themes and the significant developments in recent years: the study of strong shift equivalence (Boyle-Kim-Roush), a staple since the 1973 Annals paper of Williams; multidimensional symbolic dynamics (Aubrun-Sablik, Kass-Madden and Louidor-Marcus-Pavlov), which is enjoying a flood of interesting and interdisciplinary developments; the still developing theory of symbolic extensions and entropy structure (Downarowicz-Huczek); the emergence and development of the Bratteli-Vershik maps as a fundamental tool (Frick-Ormes); Borel dynamics (Hochman); the dimension representation (Long); cellular automata (Jadur-Nasu-Yazlle); traditional hard core coding (Jung-Lee); and connections with $C^{*}$-algebras (Krieger-Matsumoto).

The "textile systems" theory developed by Nasu remains for some purposes the most advanced machinery for analyzing the $\mathbb{Z}^{d}$ dynamics generated by endomorphisms and automorphisms of a shift of finite type. His short note on LR automorphisms, previously available only in a local publication of Mie University, is a delicate and significant example in this theory. I'm grateful that Professor Nasu has responded positively to my solicitation of the note for this volume.

Finally, I'm grateful to Kirsten Eisenträger for her review of the work of Kim and Roush on decidability questions in algebra and number theory, which grew out of their work on decidability of shift equivalence.

Editor

M. Boyle ( $\varangle)$

Department of Mathematics, University of Maryland, College Park, MD 20742-4015, USA

e-mail:mmb@math.umd.edu 